\title{
Microscopic viscosity measurement using orbital rotation in dual-beam fiber-optic trap with transverse offset
}

\author{
Mingrun Pei, Shilong Jin, Xinlin Chen $\odot$,* Tengfang Kuang, Wei Xiong, \\ Xiang Han, Guangzong Xiao $\odot$,* and Hui Luo \\ National University of Defense Technology, College of Advanced Interdisciplinary Studies, \\ Changsha, China
}

\begin{abstract}
We presented an optical system that could measure the viscosity coefficient of liquid in a micro-area. The orbital rotation of a polystyrene microsphere was realized by a dual-beam fiber-optic trap with a transverse offset. The rotation rate increased with the viscosity coefficient of the environmental medium. On this basis, the viscosity coefficients of ethanol solutions with different concentrations were measured successfully. The volume of solution samples was less than $1 \mu \mathrm{L}$. This provides a basis for the viscosity measurement of rare liquid or enchylema, which is of great significance for biological applications such as cell characteristics and reaction dynamics. (1) The Authors. Published by SPIE under a Creative Commons Attribution 4.0 Unported License. Distribution or reproduction of this work in whole or in part requires full attribution of the original publication, including its DOI. [DOI: 10.1117/1.OE.59.12.126106]
\end{abstract}

Keywords: fiber optics; optical tweezers; optical confinement and manipulation; viscosity coefficient.

Paper 20200743 received Jun. 23, 2020; accepted for publication Nov. 18, 2020; published online Dec. 22, 2020.

\section{Introduction}

The viscosity coefficient of liquid, also known as the dynamic viscosity, is a significant physical parameter for characterizing the resistance of fluid to shear motion. It has been widely used in the industrial, biological, and medical fields. However, for the viscosity measurement of rare liquids or bio-fluids, it is quite important to reduce the required volume of the sample. ${ }^{1,2}$ On a microliter scale, within liquid media, the Reynolds number is small, meaning that the forces arising from the viscosity of the fluid are dominant over its inertia. Viscosity is therefore the dominant force in all processes relying on transport, mixing, or diffusion, and hence measurement of the viscosity is paramount in understanding such systems. ${ }^{3}$ Researchers have conducted long-term and indepth research on viscosity measuring methods. Among them, the commonly used methods are falling ball, ${ }^{4}$ capillary tube method, ${ }^{5}$ and vibration measurement method. ${ }^{6}$ However, these methods are all only suitable for the measurement of the viscosity coefficient of masses of liquid, not micro-liquid or liquid in micro-areas.

Optical tweezers make measuring the viscosity in micro-areas possible. In 2005, Pesce et al. ${ }^{7}$ described a method that combined an unbiased position detector calibration procedure and frequency analysis of the Brownian motion of optically confined polystyrene microspheres. The measurement of the viscosity coefficient of pure water was realized by this method. ${ }^{8}$ However, this method could only be used to measure low or medium viscous materials, such as distilled water, bio-fluids, sucrose, glycerol solutions, and silicone oils. ${ }^{9}{ }^{10}$ In 2007, Parkin et al. ${ }^{11}$ investigated the performance and accuracy of a micro-viscometer based on rotating optical tweezers and measured the viscosity coefficient of tears. In 2013, Cooper et al. ${ }^{12}$ measured the viscosity of a fetal bovine serum using the falling ball method based on optical tweezers. However, these methods are not considered to be widely applicable because they are limited by a highly controlled beam profile or samples with special optical properties. ${ }^{13,14}$

*Address all correspondence to Xinlin Chen, xlchencs@163.com; Guangzong Xiao, xiaoguangzong@nudt.edu.cn 
In 2016, we realized the orbital rotation of the microspheres by a dual-beam fiber-optic trap with a transverse offset. ${ }^{15}$ In this paper, we present that the period of orbital rotation of the microspheres varied with the viscosity coefficient of liquid. Based on this, the viscosity coefficient of liquid in a micro-area could be measured. The method we propose not only realizes miniaturization of the optical trap system but also has a simple operation that is reliable. Our method will have a broad application in biological and chemical fields.

\section{Fundamentals}

The ray-optics can be used to calculate the trapping force when the microsphere size is much larger than the wavelength of light. ${ }^{16}$ The surface of the trapped object is divided into a finite number of small surface fractions to calculate the optical force exerted by a Gaussian beam. The direction and intensity of the ray that shines on each surface fraction can be determined according to the Gaussian beam profile. ${ }^{16}$ When a single ray hits the microsphere, the trapping force generated by this ray are divided into the scattering force component $d \mathbf{F}_{\mathrm{s}}$ and the gradient force component $d \mathbf{F}_{g}$, which are given by ${ }^{17}$

$$
\begin{aligned}
& d \mathbf{F}_{\mathrm{s}}=\frac{n_{1} q_{s}}{c} \mathbf{s} d P, \\
& d \mathbf{F}_{g}=\frac{n_{1} q_{g}}{c} \mathbf{g} d P,
\end{aligned}
$$

where the refractive index of water $n_{1}=1.333, d P$ is the light power of the ray, $\mathbf{s}$ and $\mathbf{g}$ denote the unit vectors parallel and perpendicular to the ray, respectively, and $q_{s}$ and $q_{g}$ are the efficiency factors of the optical force, which are given by ${ }^{18}$

$$
\begin{gathered}
q_{s}=1+R \cos 2 \alpha_{i}-T^{2} \frac{\cos \left(2 \alpha_{i}-2 \alpha_{r}\right)+R \cos 2 \alpha_{i}}{1+R^{2}+2 R \cos 2 \alpha_{r}} \\
q_{g}=-R \sin 2 \alpha_{i}+T^{2} \frac{\sin \left(2 \alpha_{i}-2 \alpha_{r}\right)+R \sin 2 \alpha_{i}}{1+R^{2}+2 R \cos 2 \alpha_{r}}
\end{gathered}
$$

where $\alpha_{i}$ and $\alpha_{r}$ are the angles of incidence and refraction, respectively, and $R$ and $T$ are the Fresnel reflectance and transmittance at the surface of the microsphere, respectively. For an unpolarized incident beam, the reflection and transmission coefficients are calculated by the average of $s$ and $p$ polarizations. We thus have

$$
R=\frac{1}{2}\left[\frac{\sin \left(\alpha_{i}-\alpha_{r}\right)^{2}}{\sin \left(\alpha_{i}+\alpha_{r}\right)^{2}}+\frac{\tan \left(\alpha_{i}-\alpha_{r}\right)^{2}}{\tan \left(\alpha_{i}+\alpha_{r}\right)^{2}}\right], \quad T=1-R
$$

The total optical force $\mathbf{F}_{\text {tot }}$ of the dual-beam fiber-optic trap is obtained as the sum of the force applied by two fibers. ${ }^{19}$ No torques act on the microsphere. ${ }^{17}$ Further, the O-xyz coordinate system is established as shown in Fig. 1.

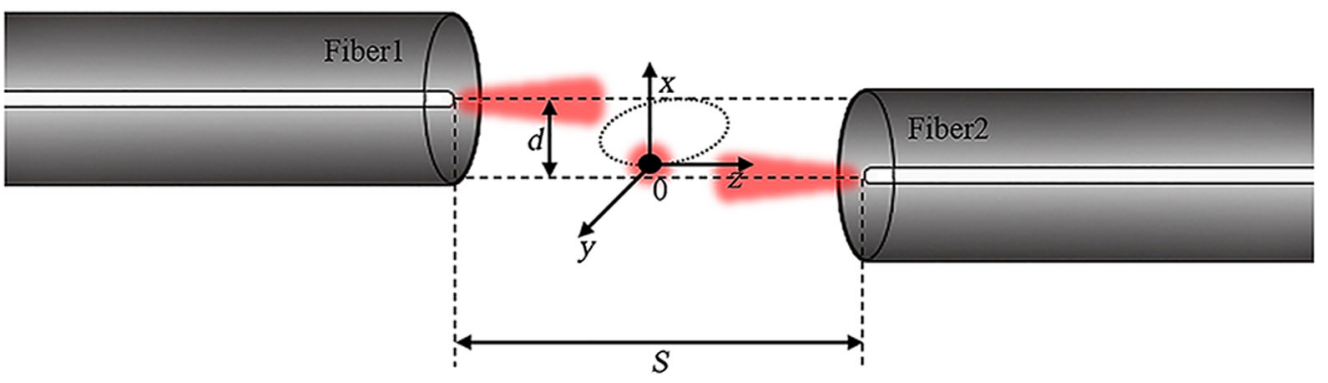

Fig. 1 Schematic of the dual-beam fiber-optic trap with transverse offset. $d$ : offset distance. 
When moving in the water, the microsphere is also affected by the viscosity resistance: ${ }^{20}$

$$
\mathbf{F}_{v}(\mathrm{t})=-6 \pi r_{0} \mathbf{v} \eta
$$

where $\eta$ is the viscosity of the surrounding medium, $r_{0}$ is the radius of the microsphere, and $v$ represents the velocity of the microsphere, which is given by

$$
\mathbf{v}(t, r)=\frac{\mathbf{F}_{\text {tot }}(r)}{6 \pi r_{0} \eta} .
$$

The program designed to simulate the trajectory of the microsphere is based on calculating its position after a small increment of time $\Delta t$. The optical forces are set as constants over the time interval $\Delta t$. The next position of the microsphere after $\Delta t$ is located by the Runge-Kutta method: ${ }^{21}$

$$
\begin{aligned}
\mathbf{k}_{1 i} & =\mathbf{v}\left(t, \mathbf{r}_{i}\right), \\
\mathbf{k}_{2 i} & =\mathbf{v}\left(t+\Delta t / 2, \mathbf{r}_{i}+\mathbf{k}_{1 i} \cdot \Delta t / 2\right), \\
\mathbf{k}_{3 i} & =\mathbf{v}\left(t+\Delta t / 2, \mathbf{r}_{i}+\mathbf{k}_{2 i} \cdot \Delta t / 2\right), \\
\mathbf{k}_{4 i} & =\mathbf{v}\left(t+\Delta t / 2, \mathbf{r}_{i}+\mathbf{k}_{3 i} \cdot \Delta t\right), \\
\mathbf{r}_{i+1} & =\mathbf{r}_{i}+\left(\mathbf{k}_{1 i}+\mathbf{k}_{2 i}+\mathbf{k}_{3 i}+\mathbf{k}_{4 i}\right) \Delta t / 6 .
\end{aligned}
$$

The magnitude and direction of the optical forces on the microsphere are recalculated for the new position, and the displacement process is repeated. The process is repeated until the desired dynamic properties are obtained.

\section{Numerical Results}

In this section, we carried out the trajectory simulation and motion frequency of the microsphere trapped in the dual-beam fiber-optical trap with a transverse offset. The fibers were separated by a width of $300 \mu \mathrm{m}$ with an offset distance of $d=9.7 \mu \mathrm{m}$. The wavelength of the trapping laser

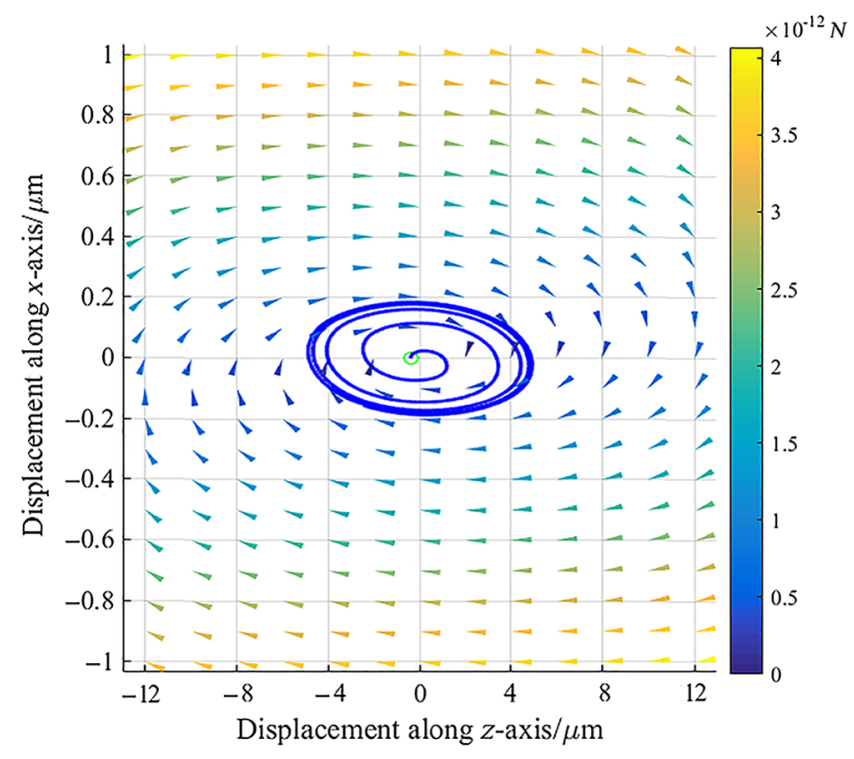

Fig. 2 The simulated trajectory of the microsphere. The colors and directions of the arrows, respectively, represent the magnitudes and directions of trapping forces. The blue solid curve denotes the trajectory of the microsphere. The green circle indicates the initial position of the microsphere. 


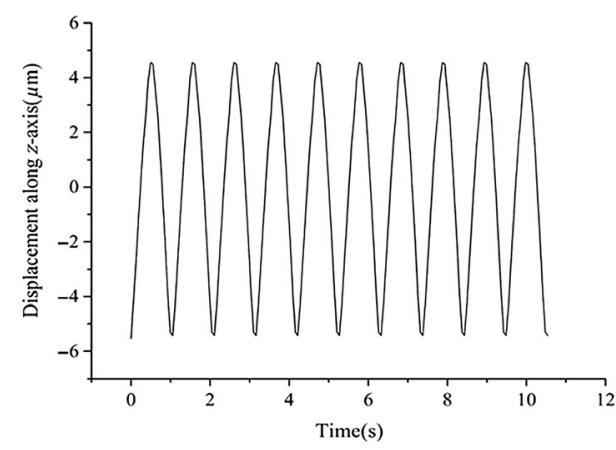

(a)

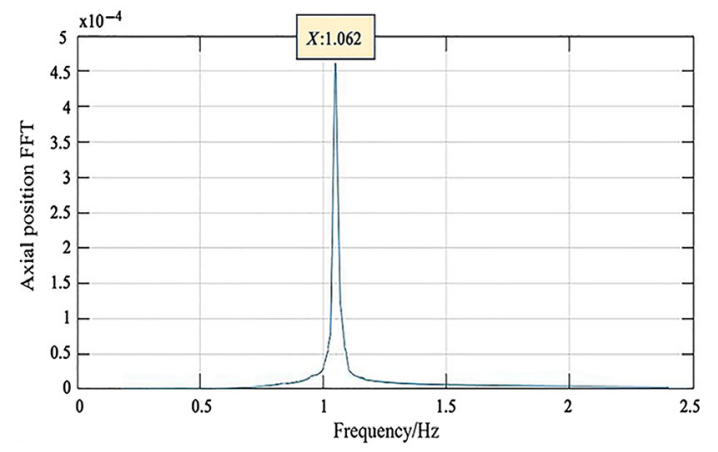

(b)

Fig. 3 (a) Vibration diagram and (b) spectrum of the microsphere in the $z$ position.

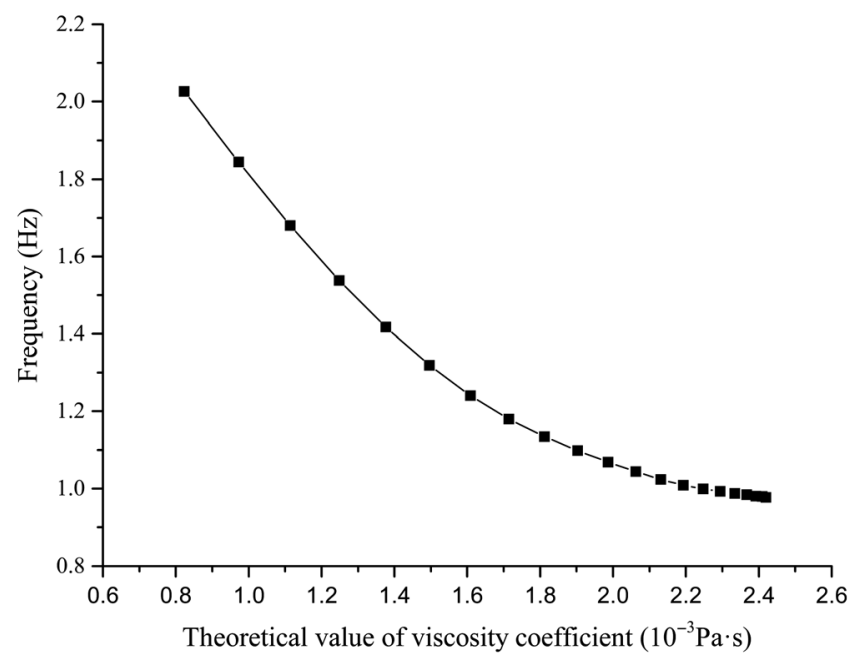

Fig. 4 Diagram of the viscosity coefficient and orbital motion frequency.

was $1064 \mathrm{~nm}$. The radius of the microspheres was $5 \mu \mathrm{m}$ with the refractive index of 1.59 . We chose deionized water (refractive index $n_{1}=1.333$, viscosity coefficient $\eta=1.005 \times$ $10^{-3} \mathrm{~Pa} \cdot \mathrm{s}$ ) as the surrounding medium.

Figure 2 shows the simulated trajectory of the microsphere. Due to the joint action of the optical trapping force and the viscous resistance, the microsphere rotates along an approximate elliptic orbit in the optical trap. ${ }^{22}$

In Fig. 3(a), we plotted the vibration waveform of the microsphere in the $z$ direction. The vibration of the microsphere was periodic, and the amplitude was stable. Figure 3(b) shows the power spectrum, which was calculated by the fast Fourier transformation. The orbital rotation frequency of the microspheres was $1.062 \mathrm{~Hz}$.

Figure 4 shows the simulated orbital rotation frequency versus the viscosity coefficient of surrounding medium. According to Eqs. (2) and (3), the velocity of the microsphere decreases with the increase of the viscosity coefficient, resulting in the decrease of the orbital rotation frequency. Finally, the viscosity of the unknown mixture could be estimated based on the orbital rotation frequency of the captured microspheres.

\section{Experiment}

The optical layout for the experiment is shown in Fig. 5. In this system, two sets of 1064-nm wavelength lasers, respectively, output two trap lights. For each optical path, a fast variable optical attenuator is used to control the laser power of individual fiber. Then, $10 \%$ of the beam 


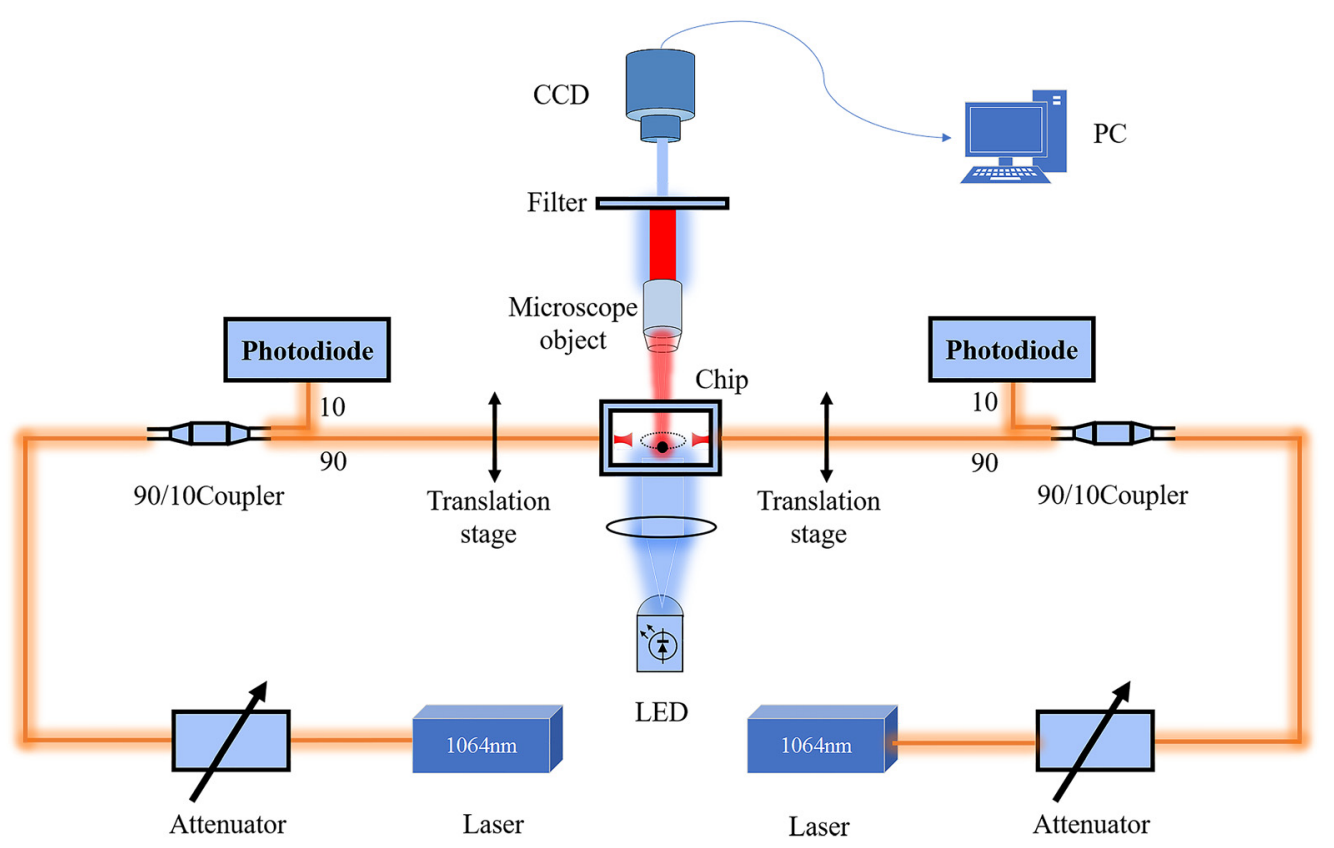

Fig. 5 Schematic diagram of the experimental instrument.

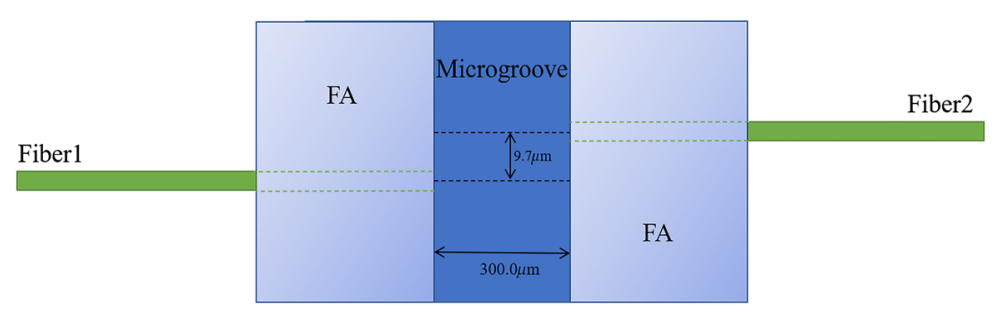

Fig. 6 Schematic diagram of the micro-chip for the optical trap.

is directed toward a photodetector, and $90 \%$ of the beam is directed to the micro-chip for the optical trap. Each fiber is attached to a translation stage to adjust the offset distance. A lightemitting diode is placed under the micro-chip for the dual-beam fiber-optic trap as the light source. The trapped microspheres in the chip are observed by a $10 \times$ microscopic objective lens and a CCD camera. A filter is placed in front of the CCD to filter out the capturing laser.

The concept diagram of the micro-chip is shown in Fig. 6. Two fibers are fixed by fiber arrays (FA). The spacing of the waveguide end is $300 \mu \mathrm{m}$, and the transverse offset is $9.7 \mu \mathrm{m}$. The size of chip is $4 \mathrm{~mm} \times 8 \mathrm{~mm} \times 1 \mathrm{~mm}$. During the experiment, the liquid sample containing microspheres is imported into the microgroove between the FA. The height and width of the microgroove are, respectively, 1 and $2 \mathrm{~mm}$. The volume of the liquid sample required in the experiment is less than $1 \mu \mathrm{L}$. The micro-chip has a good sealing property, which enables it to avoid the impact of jerks in flow.

In the experiment, we used ethanol solution at the concentrations of $10 \%$ to $50 \%$ as the liquid sample. The captured microspheres are polystyrene microspheres with a radius of $5 \mu \mathrm{m}$. The experimental phenomenon is shown in Fig. 7, in which the microsphere is rotated along an ellipse in the optical trap.

The measured rotation frequencies of the microspheres with different concentrations of alcohol are shown in Fig. 8. The orbital rotation frequency of the microsphere decreases with the increase of alcohol concentration.

The viscosity coefficient of the alcohol samples with different concentrations are given in Figs. 4 and 8. Figure 9 shows the comparison of experimental and theoretical values of the viscosity coefficient. The dots represent the viscosity coefficient measured in experiment; 
Pei et al.: Microscopic viscosity measurement using orbital rotation...

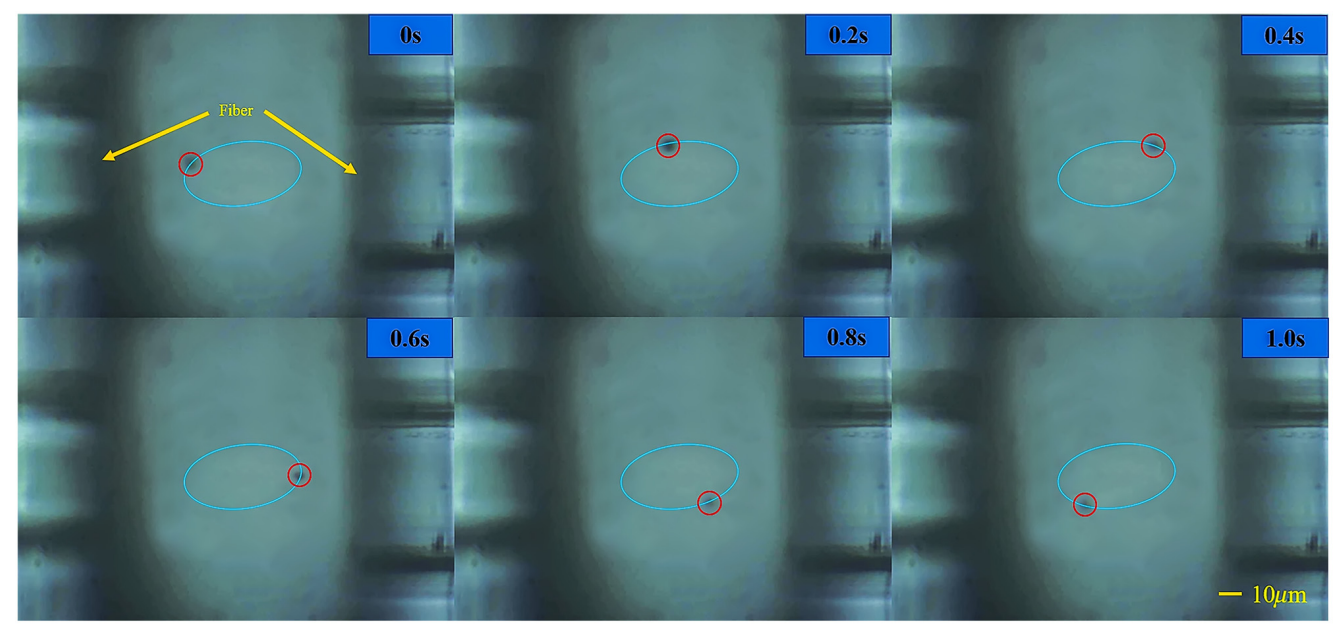

Fig. 7 Experimental phenomenon of the orbit rotation of the microsphere.

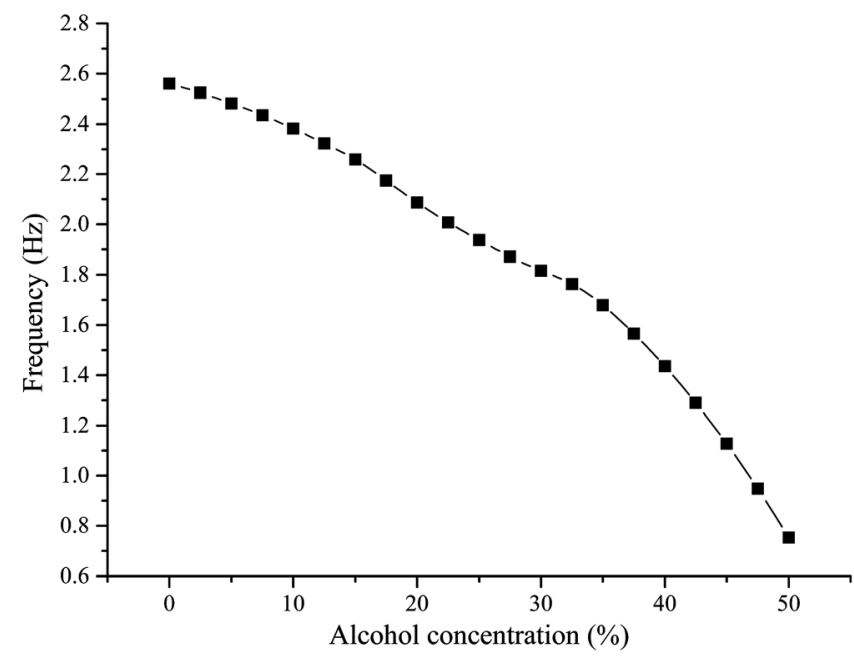

Fig. 8 The relationship between the frequency of orbital rotation and the alcohol concentration.

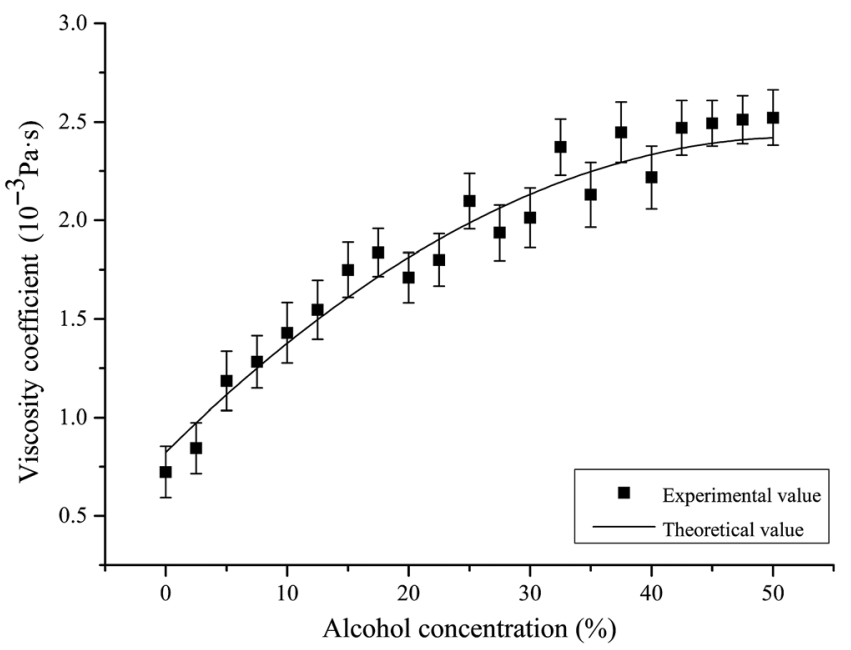

Fig. 9 Comparison of experimental and theoretical values of the viscosity coefficient. The error bar on each point represents the standard deviation of five measurements. 
Pei et al.: Microscopic viscosity measurement using orbital rotation...

the error bar on each point represents the standard deviation of five measurements. The solid curves represent theoretical values, and the squares denote the experimental results. The theoretical value of the viscosity coefficient used in Fig. 9 was measured by the falling ball method. ${ }^{23}$ Compared with it, the error of the viscosity coefficient measured by our method is less than 5\%, which can achieve accurate measurement of the viscosity coefficient of the liquid.

\section{Conclusion}

In conclusion, we realized the orbit rotation of polystyrene microspheres in a dual-beam fiberoptic trap with a transverse offset. The frequency of microspheres orbital rotation increases with the viscosity coefficient of the liquid. Then the viscosity coefficients of ethanol solutions with different concentrations were obtained by measuring the rotation frequency. The measuring error is less than $5 \%$.

Compared with other existing viscosimeter, this system results in a lower fabrication cost, requires a smaller volume of the sample, and has much higher integration. In this experiment, the required volume of the sample was less than $1 \mu \mathrm{L}$, which is conducive to the measurement of the viscosity coefficient of rare liquid in micro-areas. The volume of this viscosimeter was only $32 \mathrm{~mm}^{3}$.

The method is also expected to achieve greater development in the measurement of the viscosity coefficient of non-uniform liquid in chemistry, biology, microrheology, and other microscopic fields, such as corrosive liquids and cell SAP. In the future, an optical tweezers chip is expected to replace the viscometer as a new tool for measuring the viscosity coefficient.

\section{Acknowledgments}

This work was supported by the National Natural Science Foundation of China (Nos. 11904405 and 61975237). The authors declare no conflicts of interest.

\section{References}

1. S. Keen et al., "Multipoint viscosity measurements in microfluidic channels using optical tweezers," Lab Chip 9, 2059-5062 (2009).

2. A. Sasso and G. Pesce, "Optical tweezers calibration: a quantitative tool for local viscosity investigation (Invited Paper)," Proc. SPIE 5830, 368-378 (2005).

3. M. A. Antar and M. A. I. El-Shaarawi, "Effect of viscosity ratio, spin and Reynolds number on the flow characteristics about a liquid sphere in a gas stream," Int. J. Numer. Methods Heat Fluid Flow 12(7), 800-816 (2012).

4. A. W. Francis, "Wall effect in falling ball method for viscosity," Physics 4(11), 403-406 (1933).

5. R. Wagner and J. Russell, "Capillary-tube viscometer," Anal. Chem. 20(2), 151-155 (1948).

6. V. Primachenko et al., "Postservice analysis of zirconia nonswirl nozzles for intermediate ladles of a continuous casting machine," Refract. Ind. Ceram. 44(1), 1-3 (2003).

7. G. Pesce, A. Sasso, and S. Fusco, "Viscosity measurements on micron-size scale using optical tweezers," Sci. Instrum. Rev. 76, 115105 (2005).

8. Y. Zhang et al., "Measurement of the microscopic viscosities of microfluids with a dynamic optical tweezers system," Laser Phys. 24(6), 065601 (2014).

9. J. Wang et al., "Measurement of viscosity using a long-period fiber-grating-based viscometer," Adv. Mater. Res. 723, 420-427 (2013).

10. L. Tanner, "The measurement of viscosity by optical techniques applied to a falling liquid film," J. Phys. E Sci. Instrum. 9(11), 967-973 (1976).

11. S. Parkin et al., "Picolitre viscometry using optically rotated particles," Phys. Rev. E Stat. Nonlinear Soft Matter Phys. 76(4), 041507 (2007).

12. J. P. Cooper et al., "Application of laser trap as a viscometer," in Eur. Conf. Biomed. Opt., pp. 87-97 (2013). 
13. S. Roy et al., "Aquatic model for engine oil degradation by rhamnolipid producing Nocardiopsis," VITSISB Biotech. 3(5), 153-164 (2015).

14. A. Pralle et al., "Local viscosity probed by photonic force microscopy," Appl. Phys. A. 66(7), S71-S73 (1998).

15. X. Chen et al., "Characteristics of the orbital rotation in dual-beam fiber-optic trap with transverse offset," Opt. Express 24(15), 16952-16960 (2016).

16. G. Xiao et al., "Orbital rotation of trapped particle in a transversely misaligned dual-fiber optical trap," IEEE Photonics 8(1), 1-8 (2016).

17. A. Ashkin, "Forces of a single-beam gradient laser trap on a dielectric sphere in the ray optics regime," Biophysics 61(2), 569-582 (1992).

18. N. Watanabe and K. Taguchi, "Theoretical study of optical vibration and circulation of a microsphere," Key Eng. Mater. 516, 563-568 (2012).

19. E. Sidick, S. D. Collins, and A. Knoesen, "Trapping forces in a multiple-beam fiber-optic trap," Appl. Opt. 36(25), 6423-6433 (1997).

20. J. Foo, K. Liu, and V. Chan, "Viscous drag of deformed vesicles in optical trap," Exp. Simul. AICHE J. 50(1), 249-254 (2004).

21. D. Erenso et al., "Formation of synthetic structures with micron size silica beads using optical tweezer," J. Mod. Opt. 54(10), 1529-1536 (2007).

22. X. Chen et al., "Dynamics analysis of microsphere in a dual-beam fiberoptic trap with transverse offset," Opt. Express 24(7), 7575-7584 (2016).

23. S. S. Ali, Fundamental Interactions and Physical Properties of Starch, Poly Vinyl Alcohol and Montmorillonite Clay Based Nanocomposites Prepared Using Solution Mixing and Melt Extrusion, Kansas State University (2010).

Mingrun Pei is an MS candidate at the National University of Defense Technology. He received his BS degree in physics from the National University of Defense Technology in 2015. His current research interests focus on the optical trap.

Shilong Jin received his PhD from the National University of Defense Technology in 2005. Currently, he is a professor at the National University of Defense Technology.

Xinlin Chen received his PhD from the National University of Defense Technology in 2018. Currently, he is a lecturer at the National University of Defense Technology. His research focuses on the optical trap.

Tengfang Kuang is a $\mathrm{PhD}$ student at the National University of Defense Technology. He received his MS degree in physics from the National University of Defense Technology in 2019. He is working on optical trap and opto-electronics systems.

Wei Xiong received his PhD from the National University of Defense Technology in 2019. Currently, he is a lecturer at the National University of Defense Technology. He is working on the optical trap and optical inspection.

Xiang Han received his PhD from the National University of Defense Technology in 2017. Currently, he is a lecturer at the National University of Defense Technology. He is working on the optical trap.

Guangzong Xiao received his PhD from the National University of Defense Technology in 2011. Currently, he is a professor at the National University of Defense Technology. His research focuses on the optical trap.

Hui Luo received his PhD from the National University of Defense Technology in 1997. Currently, he is a professor at the National University of Defense Technology. 